\title{
Masquelet induced membrane technique for treatment of rat chronic osteomyelitis
}

\author{
TAO CUI $^{1^{*}}$, JUNPENG LI $^{2}$, PING ZHEN ${ }^{3}$, QIUMING GAO $^{3}$, XIAOHAI FAN $^{3}$ and CHUANGBING LI ${ }^{3}$ \\ ${ }^{1}$ Department of Orthopaedics, Cangzhou Central Hospital, Cangzhou, Hebei 061000; \\ ${ }^{2}$ Department of Surgery (I), Armed Police Engineering University Hospital, Xian, Shaanxi 710000; \\ ${ }^{3}$ Department of Traumatic Orthopedics, Lanzhou General Hospital of CPLA, Lanzhou, Gansu 730050, P.R. China
}

Received January 29, 2018; Accepted July 18, 2018

DOI: $10.3892 / \mathrm{etm} .2018 .6573$

\begin{abstract}
The application value of Masquelet induced membrane technique for the treatment of rat chronic osteomyelitis was explored. A total of 180 male Sprague-Dawley (SD) rats were randomly divided into sham operation group (control group), chronic osteomyelitis model group (model group) and Masquelet induced membrane therapy + chronic osteomyelitis model group (observation group); 60 rats in each group. A rat model of traumatic osteomyelitis was established using a modified blunt trauma method in model and observation group. All rats in observation group were treated with membrane induction technology while in the control group they were not. Postoperative complications, bone healing time and functional status of the affected limbs were recorded. After surgery, no obvious perioperative complications occurred in most of the rats, except that 5 rats experienced partial necrosis at the edge of the flap, and 3 experienced superficial infection around the incision site in the ilium. Primary bone healing was achieved in 50 rats with an average healing time of 15 weeks. Among them, 38 rats restored weight-bearing function after 20 weeks. Seven rats experienced infection after surgery, but complete bone healing was achieved after treatment with induced membrane technique again. Following treatment with induced membrane technique, the rat body weight, body temperature and the number of bacteria in the wound decreased over time. In conclusion, Masquelet induced membrane technique is effective for the treatment of large bone defects in rats after traumatic osteomyelitis. This technique significantly shortens the treatment cycle. The surgical procedure is simple, and the incidence of postoperative complications is low.
\end{abstract}

Correspondence to: Dr Chuangbing Li, Department of Traumatic Orthopedics, Lanzhou General Hospital of CPLA, 333 South Binhe Road, Lanzhou, Gansu 730050, P.R. China

E-mail: bby66z@163.com

*Contributed equally

Key words: induced membrane technique, osteomyelitis, rat, tibia fracture, femoral fracture

\section{Introduction}

Chronic osteomyelitis is a common surgical disease that can be divided into three categories: spread of local infection due to incomplete debridement of an infected open wound, chronic inflammation developed from acute osteomyelitis, and iatrogenic infection $(1,2)$. Pathogenic bacteria can develop drug resistance due to long-term use of antibiotics for serious local injuries, resulting in delayed healing (3). The treatment of chronic osteomyelitis is difficult and the procedure is complex. Control of infection and repair of bone defects are main focuses of the treatment of chronic osteomyelitis. Inappropriate treatments often lead to recurrent local inflammation and serious complications such as muscle atrophy or even amputation (4). Traditional treatment methods, such as bone graft surgery, are more effective in patients with small bone defects than in patients with large bone defects such as femoral and tibia defects due to limited resources of donor bone (5-7). Skeletal traction is commonly used for the treatment of large bone defects in clinic. However, the operation procedure is complicated and multiple complications may occur (8). Induced membrane technique is a new and popular treatment method that has been reported to be commonly used for the treatment of large bone defects in clinic. Induced membrane technique is a 2-step procedure: formation of a membrane at the site of bone loss induced by putting a transitory poly(methyl methacrylate) spacer, and integration therein of autologous cancellous bone graft collected from other parts of the patient's body. The procedure is simple and efficacy is high $(9,10)$. The treatment with induced membrane technique focuses on two key aspects: control of infection in the bone defects and reconstruction of the damaged bone (11). A study found that membrane induction technology in the treatment of chronic osteomyelitis in rabbits can reduce the incidence of infection and inflammatory cell concentrations (12). Membrane induction technology for the treatment of patients with clinical chronic osteomyelitis can shorten bone healing time and reduce the incidence of postoperative complications (13). At present, studies on the treatment of chronic osteomyelitis with induced membrane technique mainly focus on therapeutic efficacy. There is no study on animal models which may facilitate an in-depth investigation. In this study, the induced membrane technique was used to treat rats with chronic osteomyelitis. Treatment efficacy and changes of clinical indications were recorded. The 
application value of membrane induction technology was evaluated using indexes including serum leukocyte count, C-reactive protein (CRP) and tumor necrosis factor- $\alpha$ (TNF- $\alpha)$. Our study provided reference for the treatment of chronic osteomyelitis with induced membrane technique.

\section{Materials and methods}

Animals. A total of 180 healthy male Sprague-Dawley (SD) rats, weighing 190-220 g, were purchased from Beyotime Institute of Biotechnology (Shanghai, China) and fed under standard conditions. The rats were randomly divided into sham operation group (control group), chronic osteomyelitis model group (model group) and Masquelet induced membrane therapy + chronic osteomyelitis model group (observation group); 60 rats in each group. A rat model of traumatic osteomyelitis was established using a modified blunt trauma method in model and observation group. All rats in observation group were treated with membrane induction technology, while those in control group were not. The present study was approved by the Ethics Committee of the Cangzhou Central Hospital (Cangzhou, China).

Establishment of osteomyelitis model. A rat traumatic osteomyelitis model was established using a modified rat blunt trauma method (12). SD rats were fed for 1 week under standard conditions. Rats were fasted for $12 \mathrm{~h}$ and subperitoneal anesthesia was performed with $10 \%$ chloral hydrate at a dose of $10 \mathrm{mg} / 100 \mathrm{~g}$. Rats were then fixed on the operation table. Operation area was shaved and disinfected and a 7-gauge needle was inserted into the bone marrow cavity from the anterior middle. Sodium morrhuate $(0.1 \mathrm{ml}$ of $5 \%), 0.3 \mathrm{ml}$ of Staphylococcus aureus suspension and $0.3 \mathrm{ml}$ of normal saline were injected through the needle. After 2 weeks, local swelling and pus were observed. X-ray scanning was performed to show periosteal proliferation, cortical thickening and formation of sequestrum in the long bone.

Treatment with induced membrane technique. Treatment with induced membrane technique was performed in 2 steps and all rats were under general anesthesia. In the first step, radical debridement was performed and implantation of antibiotics and bone cement was performed. Briefly, debridement was performed to expose normal cortical bone interface. Bone defect was fixed using intramedullary nails. Vancomycin and PMMA bone cement was mixed at a ratio of 1:20 and put into bone defect to connect both ends. Then the incision was closed. If inflammation occurred within 6-8 weeks the first step was repeated. In step 2, bone defect was reconstructed by cancellous bone grafting. Briefly, an incision was made to expose the induced membrane formed around the defect. Membrane was cut longitudinally and then bone cement was removed carefully. The defect was filled with morselized cancellous bone graft. The incision was closed, washed with saline and disinfected with iodophor.

Observed indicators. After the rat model was established, the following indicators were observed or measured: the levels of wound swelling and pus formation, the rat body temperature recorded at 10:00 a.m. daily from day 1 to day 8 after surgery, the rat body weight recorded on the day of surgery as well as day 7 and day 14 after surgery, and the number of bacteria in the
Table I. Treatment of the complications of chronic osteomyelitis in rats with induced membrane technique.

\begin{tabular}{lc} 
Complication type & No. of cases $(\%)$ \\
\hline Partial necrosis at edge of the flap & $5(8.33)$ \\
$\begin{array}{l}\text { Superficial infection around the incision } \\
\text { site in the ilium }\end{array}$ & $3(5.00)$ \\
No complications & $52(86.67)$
\end{tabular}

Table II. Outcomes of the treatment of chronic osteomyelitis in rats with induced membrane technique.

\begin{tabular}{lc}
\hline Treatment outcomes & $\begin{array}{c}\text { Cases }(\%) \text { or } \\
\text { mean } \pm \text { SD }\end{array}$ \\
\hline Primary bone healing & $50(83.33)$ \\
Healing time (weeks) & $15 \pm 1.56$ \\
Restoration of weight-bearing function & $38(63.33)$ \\
Time for restoration of weight-bearing & $20 \pm 1.80$ \\
function (weeks) & \\
Postoperative infection & $7(11.67)$ \\
\hline
\end{tabular}

wound. Blood was collected from tail blood to prepare serum. Serum TNF- $\alpha$ was detected using an ELISA kit (Shanghai Fanke Biotechnology Co., Ltd., Shanghai, China). White blood cells were counted using a blood cell analyzer (MEK-822K) (Nihon Kohden, Tokyo, Japan), and CRP level was measured using an automatic biochemical analyzer (Hitachi, Ltd., Tokyo, Japan) (14).

Statistical analysis. SPSS 13.0 software (SPSS, Inc., Chicago, IL, USA) was used for the statistical analyses of the data. Data were presented as mean \pm SD. Quantitative data were processed using analysis of variance or t-test. SNK-q test was used for pairwise comparison. Spearman's correlation analysis was used to determine the correlation between body temperature and days after treatment. All P-values represent bilateral probability, and the level of significance $\alpha$ is 0.05 .

\section{Results}

Basic information of rat. The 180 rats were divided into 3 groups and each group included 10 cases of tibia fracture, femoral fracture, humerus fracture, ulnar and radial fractures, diaphysis fracture and metaphyseal fracture, respectively. The average age of rats in the three groups was $8.20 \pm 0.35,8.25 \pm 0.45$ and $8.61 \pm 0.51$ weeks, respectively $(\mathrm{P}>0.05)$, and the average body weight was $210.51 \pm 10.62$, $209 \pm 9.89$ and $211.52 \pm 11.01 \mathrm{~g}$, respectively $(\mathrm{P}>0.05)$.

Complications of induced membrane technique. In observation group, $5(8.33 \%)$ rats developed flap marginal necrosis, and $3(5.00 \%)$ rats developed superficial infection of patella. In model group, $14(23.33 \%)$ rats had flap marginal necrosis, and $12(20.00 \%)$ rats showed superficial infection of patella. 
Table III. Number of bacteria in the surgical wound before and after treatment with induced membrane technique $\left(\mathrm{n} / \mathrm{mm}^{3}\right.$, mean $\pm \mathrm{SD})$.

\begin{tabular}{lccccc}
\hline & \multirow{2}{*}{$\begin{array}{c}\text { Before } \\
\text { Indicator }\end{array}$} & \multicolumn{4}{c}{ After treatment } \\
\cline { 3 - 6 } & treatment & Day 1 & Day 3 & Day 5 & Day 7 \\
\hline Bacteria count & $20,618.71 \pm 865.25$ & $1,120.63 \pm 121.28$ & $218.51 \pm 30.16$ & $50.44 \pm 11.92$ & $10.28 \pm 1.26$ \\
\hline
\end{tabular}

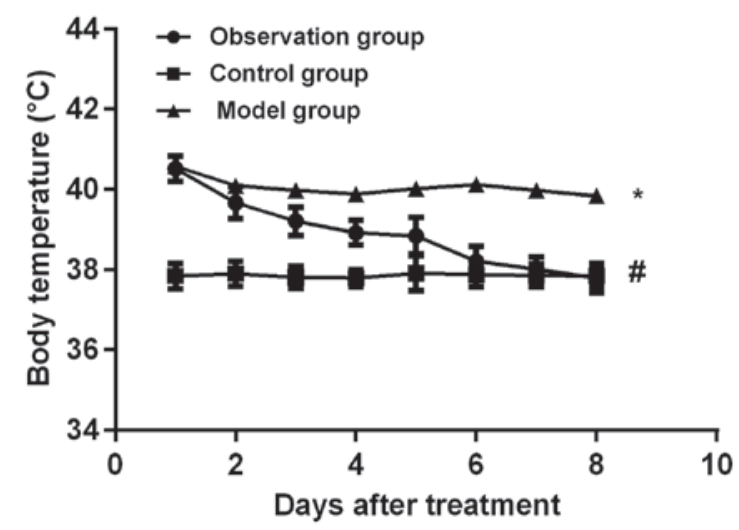

Figure 1. Body temperature changes of rat models in three groups. The rat body temperatures from day 1 to day 8 post-treatment are shown. The body temperature decrease was correlated with the number of days after treatment $(\mathrm{r}=0.976, \mathrm{P}<0.001)$. As the treatment time increased, the rats gradually returned to normal body temperature. There was no significant correlation between the body temperature and treatment days in the control and model group $(\mathrm{r}=0.098$, $\mathrm{P}>0.05)$. At day 7 after treatment, the body temperature of the rats in the observation group was lower than that in the model group $(\mathrm{P}<0.05)$. ${ }^{*} \mathrm{P}<0.05$, compared with the control group. ${ }^{\#} \mathrm{P}<0.05$, compared with the model group.

Significant differences were found between the two groups $(\mathrm{P}<0.05)$ (Table I).

Outcomes of treatment with induced membrane technique. Primary bone healing was achieved in $50(83.33 \%)$ rats with an average healing time of $15 \pm 1.56$ weeks. Among them, 38 $(76 \%)$ rats restored weight-bearing function after 20 weeks. Seven $(11.67 \%)$ rats experienced infection after surgery, but complete bone healing was achieved after treatment with induced membrane technique again (Table II). Number of bacteria in the surgical wound before and after treatment with induced membrane technique was shown in (Table III).

Rat body temperature and weight after treatment with induced membrane technique. The body temperature of rats from day 1 to day 8 after surgery was compared among the three groups. The body temperature of rats in the observation group gradually decreased. The decrease in body temperature was correlated with the number of days after treatment $(\mathrm{r}=0.976, \mathrm{P}<0.001)$. With the increase of treatment time normal body temperature gradually returned. There was no significant difference in body temperature and treatment time between the control and model group ( $\mathrm{r}=0.098, \mathrm{P}>0.05)$. On the 7 th day after surgery, the body temperature in observation group was lower than that in model group $(\mathrm{P}<0.05)$ (Fig. 1). On the 1st, 7th and 14th day after the application of membrane induction technique, the body weight of rats in the observation and model group gradually decreased

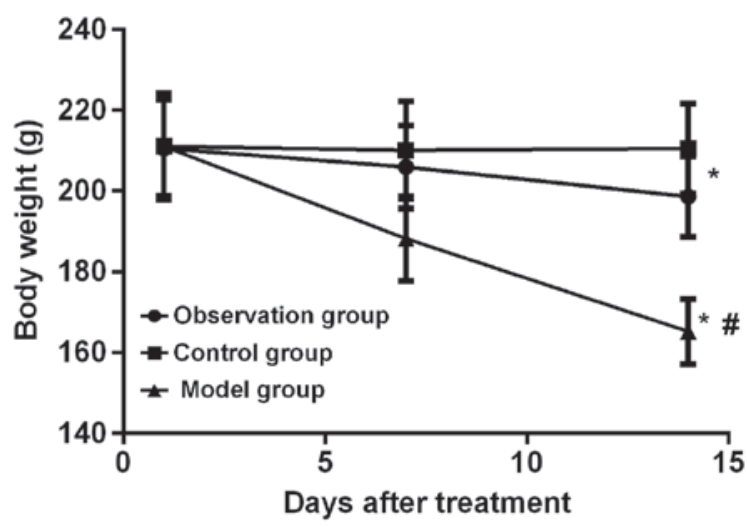

Figure 2. Body weight changes of rat models in three groups. The body weights at day 0,7 and 14 post-treatment are shown. Body weights decreased gradually in observation and model group $(\mathrm{F}=8.916, \mathrm{P}=0.005 ; \mathrm{F}=12.021$, $\mathrm{P}<0.001)$. There was no significant change in body weight in the control group $(\mathrm{P}>0.05)$. On the 14th day, the body weight of rats in the observation group was higher than that in the model group $(\mathrm{P}<0.05)$, and the weight in the control group was higher than that in the observation group $(\mathrm{P}<0.05)$. ${ }^{*} \mathrm{P}<0.05$, compared with the control group. ${ }^{\#} \mathrm{P}<0.05$, compared with the model group.

$(\mathrm{F}=8.916, \mathrm{P}=0.005 ; \mathrm{F}=12.021, \mathrm{P}<0.001)$. There was no significant change in body weight in control group $(\mathrm{P}>0.05)$. On the 14th day, the weight of the rats in the observation group was higher than that of the rats in the model group $(\mathrm{P}<0.05)$, and the weight of the rats in control group was higher than that in observation group $(\mathrm{P}<0.05)$ (Fig. 2).

Changes of serum WBC, TNF- $\alpha$ and CRP levels in rats at different time-points. During the whole course of treatment, serum WBC, TNF- $\alpha$ and CRP levels in the model group were significantly higher than those in control group $(\mathrm{P}<0.05)$. Compared with those in model group, WBC, TNF- $\alpha$ and CRP levels in observation group significantly decreased after treatment $(\mathrm{P}<0.05)$ (Figs. 3-5).

\section{Discussion}

Chronic osteomyelitis, as a major challenge to orthopedic surgery, is hard to treat (15). Staphylococcus aureus is the most common pathogen found in chronic osteomyelitis. Radical debridement by surgery is a crucial step where particular attention should be paid because large bone defects are created following radical debridement (16). Therefore, reconstruction of bone defects is another key challenge in treating chronic osteomyelitis. Concentration of antibiotics under systemic medication is too low to effectively kill bacteria at chronic osteomyelitis lesion due to poor local blood circulation (17). 


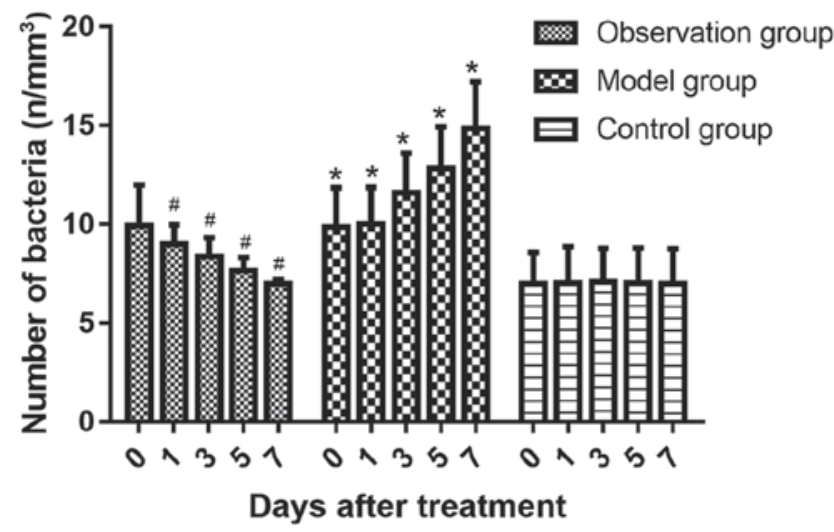

Figure 3. Comparison of serum WBC levels $\left(10^{9} / 1\right)$ at different time-points in rats. Compared with that in the control group, the serum WBC in the model group increased significantly $(\mathrm{P}<0.05)$, and the $\mathrm{WBC}$ content in the observation group decreased significantly after treatment compared with the model group $(\mathrm{P}<0.05)$. ${ }^{*} \mathrm{P}<0.05$, compared with the control group. ${ }^{*} \mathrm{P}<0.05$, compared with model group.

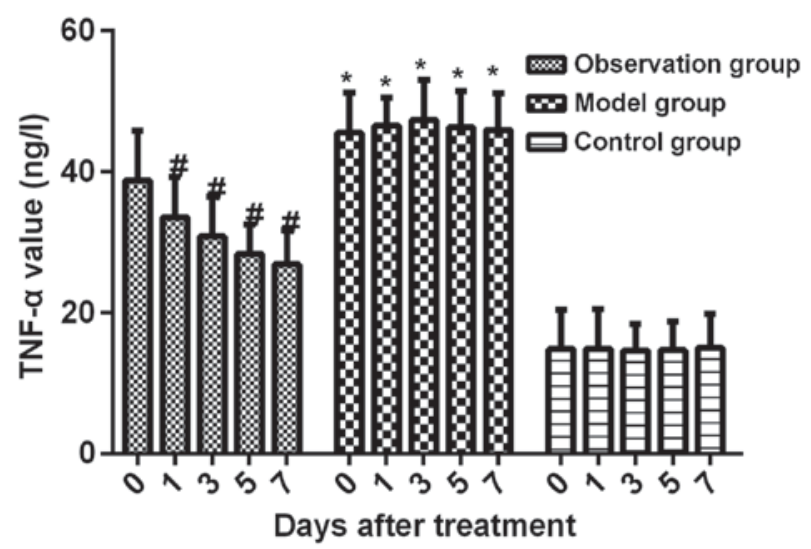

Figure 4. Comparison of serum TNF- $\alpha$ levels (ng/l) at different time-points in rats. Compared with that in the control group, the serum TNF- $\alpha$ in the model group increased significantly $(\mathrm{P}<0.05)$, and the TNF- $\alpha$ content in the observation group decreased significantly after treatment compared with that in the model group $(\mathrm{P}<0.05)$. "P<0.05, compared with the control group. ${ }^{\text {"}} \mathrm{P}<0.05$, compared with the model group. TNF- $\alpha$, tumor necrosis factor- $\alpha$.

Traditional treatment methods, such as open bone grafting, vacuum sealing drainage and antibiotic irrigation-perfusion, have a series of issues including complicated procedures, poor treatment outcomes, resulting in more complications, prolonged treatment time, and poor patient acceptance (18-21).

Induced membrane technique was first described by Masquelet in treatment of chronic osteomyelitis (5). The treatment process consists of two relatively independent surgical stages: the first step includes debridement and implantation of antibiotic bone cement in the bone defect; in the second step, membrane structure formation induction in the bone defect and bone reconstruction by autologous cancellous bone grafting are performed. In the first step, antibiotics and bone cement are mixed. After application, the antibiotics are gradually released to increase local drug concentration in chronic osteomyelitis lesions and thereby enhancing the treatment efficacy. Bone cement protects the damaged bone tissue, thereby reducing the risk of a bone fracture following radical debridement. In addition, self-solidification of antibiotic bone cement in the body allows

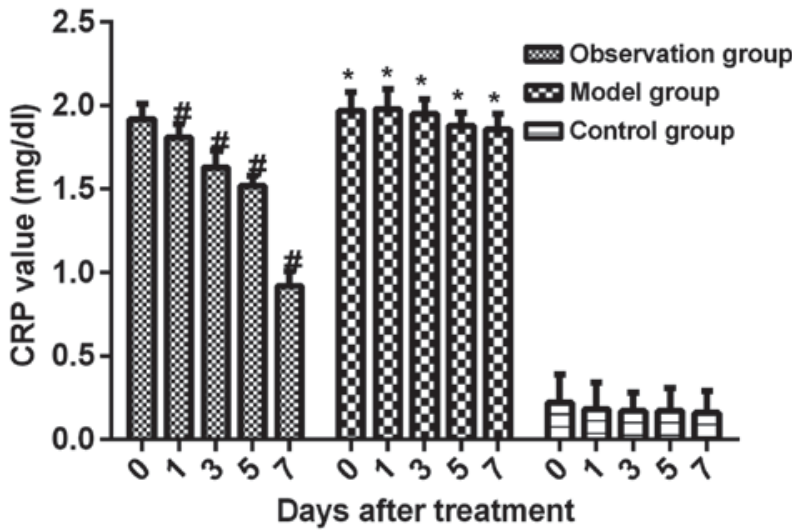

Figure 5. Comparison of serum CRP levels $(\mathrm{mg} / \mathrm{dl})$ at different time-points in rats. Compared with that in the control group, the serum CRP in the model group increased significantly $(\mathrm{P}<0.05)$, and the CRP content in the observation group decreased significantly after treatment compared with that in the model group $(\mathrm{P}<0.05)$. ${ }^{*} \mathrm{P}<0.05$, compared with the control group. ${ }^{\#} \mathrm{P}<0.05$, compared with the model group. CRP, C-reactive protein.

for high local drug concentration around bone defects. Induced membrane technique can be used to treat chronic osteomyelitis resulted from a variety of causes, due to the simple surgical procedures and satisfactory clinical efficacy. Various studies on clinical treatment of osteomyelitis using induced membrane technique have been reported and satisfactory outcomes have been observed $(22,23)$. Pelissier et al (22) used the induced membrane technique in rabbits to study the expression of various cytokines in the process of bone formation. Aho et al (23) performed membrane induction surgery on patients, and analyzed the components of induced membrane under different conditions.

In this study, traumatic osteomyelitis rat models were established and treatment with induced membrane technique was performed. Results showed that 50 of 60 rats achieved primary bone healing with a healing time of only 15 weeks, and 38 rats restored weight-bearing function. Seven rats experienced infection after surgery probably due to incomplete debridement in the first stage, but complete bone healing was achieved after treatment with induced membrane technique for the second time. The induced membrane technique treatment method is superior to traditional treatment methods in terms of number of surgeries, treatment efficacy, hospitalization expenses, complications, and recovery time (24-26). Masquelet and Begue reported that all 35 patients with bone defects in their study achieved radiographic healing following treatment with induced membrane technique (5). Another study also reported that $90 \%$ of patients achieved bone union (27).

It was found in this study that rat weight, body temperature and number of bacteria in the wound decreased over time following treatment with induced membrane technique. Weight loss may be caused by poor appetite resulted from stress during model establishment and treatment. The decreases in rat body temperature and number of bacteria in the wound suggested that local high concentration of antibiotics was achieved around bone defect after implantation of antibiotic bone cement, which effectively inhibited the growth of various bacteria and reduced the incidence of postoperative infection.

In conclusion, through establishment of rat chronic osteomyelitis models followed by treatment with induced membrane 
technique, we observed a high rate of bone union with low incidence of postoperative complications. In addition, the weight-bearing function of the affected limb was restored. Our study provided reference for the use of the induced membrane technique for the treatment of chronic osteomyelitis.

\section{Acknowledgements}

Not applicable.

\section{Funding}

No funding was received.

\section{Availability of data and materials}

The datasets used and/or analyzed during the current study are available from the corresponding author on reasonable request.

\section{Authors' contributions}

TC and JL were major contributors in writing the manuscript, they designed the methods and conceived the idea of this study. TC, JL and PZ were involved in the follow up of patients. TC, $\mathrm{JL}$ and QG were responsible for the collection of the data. TC and XF participated in the analysis and discussion of the data. CL was also involved in the conception of the study. All authors read and approved the final manuscript.

\section{Ethics approval and consent to participate}

This study was approved by the Ethics Committee of the Cangzhou Central Hospital (Cangzhou, China).

\section{Patient consent for publication}

Not applicable.

\section{Competing interests}

The authors declare that they have no competing interests.

\section{References}

1. Wang X, Yu S, Sun D, Fu J, Wang S, Huang K and Xie Z: Current data on extremities chronic osteomyelitis in southwest China: Epidemiology, microbiology and therapeutic consequences. Sci Rep 7: 16251, 2017.

2. Ferrando A, Part J and Baeza J: Treatment of cavitary bone defects in chronic osteomyelitis: Biogactive glass S53P4 vs. calcium sulphate antibiotic beads. J Bone Jt Infect 2: 194-201, 2017.

3. Kabore C, Wouters A, Frippiat F and Gillet P: Management of chronic osteomyelitis by long-term antibiotic suppression. Rev Med Liege 72: 363-368, 2017 (In French).

4. Geurts J, Hohnen A, Vranken T and Moh P: Treatment strategies for chronic osteomyelitis in low- and middle-income countries: Systematic review. Trop Med Int Health 22: 1054-1062, 2017.

5. Masquelet AC and Begue T: The concept of induced membrane for reconstruction of long bone defects. Orthop Clin North Am 41: 27-37, 2010.

6. Rousset M, Walle M, Cambou L, Mansour M, Samba A, Pereira B Ghanem I and Canavese F: Chronic infection and infected non-union of the long bones in paediatric patients: Preliminary results of bone versus beta-tricalcium phosphate grafting after induced membrane formation. Int Orthop 42: 385-393, 2018.
7. Azi ML, de Almeida Teixeira AA, Cotias RB, Joeris A and Kfuri Junior M: Bone union with an in situ spacer after the first stage of the induced membrane technique. Injury 48 (Suppl 4): S17-S20, 2017.

8. Masquelet AC: Induced membrane technique: Pearls and pitfalls. J Orthop Trauma 31 (Suppl 5): S36-S38, 2017.

9. Konda SR, Gage M, Fisher N and Egol KA: Segmental bone defect treated with the induced membrane technique. J Orthop Trauma 31 (Suppl 3): S21-S22, 2017.

10. Han W, Shen J, Wu H, Yu S, Fu J and Xie Z: Induced membrane technique: Advances in the management of bone defects. Int J Surg 42: 110-116, 2017.

11. Lan XJ: Intravenous antibiotic perfusion in chronic osteomyelitis. Zhonghua Hu Li Za Zhi 19: 337-338, 1984 (In Chinese).

12. Avdeeva EY, Slizovsky GV, Skorokhodova MG, Fomina TI Zorkaltsev MA, Zavadovskaya VD, Krasnov EA, Ivanov VV and Stepanov MY: Experimental simulation of traumatic osteomyelitis in rats. Bull Exp Biol Med 161: 137-140, 2016.

13. Argenta LC and Morykwas MJ: Vacuum-assisted closure: a new method for wound control and treatment: Clinical experience. Ann Plast Surg 38: 563-576, discussion 577, 1997.

14. Henry SL: Discussion: The efficacy of perforator flaps in the treatment of chronic osteomyelitis. Plast Reconstr Surg 140: 189-191, 2017.

15. Kinik H and Karaduman M: Cierny-Mader type III chronic osteomyelitis: The results of patients treated with debridement, irrigation, vancomycin beads and systemic antibiotics. Int Orthop 32: 551-558, 2008

16. Hong JPJ, Goh TLH, Choi DH, Kim JJ and Suh HS: The efficacy of perforator flaps in the treatment of chronic osteomyelitis. Plast Reconstr Surg 140: 179-188, 2017.

17. Narayana Kurup JK, Singasani R and Mohanty SP: Rare case of disseminated rhinosporidiosis with chronic osteomyelitis of the calcaneum treated by a simple technique of negative pressure wound therapy. BMJ Case Rep 2017: bcr-2017-221786, 2017.

18. Stanger KM, Albert F, Kneser U, Bogdan C and Horch RE: Management of chronic osteomyelitis of the tibia with life-threatening complications under negative pressure wound therapy and isolation of Helcococcus kunzii. Int Wound J 12: 443-446, 2015.

19. Peng B, Song CY, Jin HT, Xiao LW and Tong PJ: Clinical diagnosis and treatment of chronic osteomyelitis. Zhongguo $\mathrm{Gu}$ Shang 28: 870-873, 2015 (In Chinese).

20. Jiang N, Ma YF, Jiang Y, Zhao XQ, Xie GP, Hu YJ, Qin CH and Yu B: Clinical characteristics and treatment of extremity chronic osteomyelitis in southern China: A retrospective analysis of 394 consecutive patients. Medicine (Baltimore) 94: e1874, 2015.

21. Gokalp MA, Guner S, Ceylan MF, Doğan A and Sebik A: Results of treatment of chronic osteomyelitis by 'gutter procedure and muscle flap transposition operation'. Eur J Orthop Surg Traumatol 24: 415-419, 2014.

22. Pelissier P, Masquelet AC, Bareille R, Pelissier SM and Amedee J: Induced membranes secrete growth factors including vascular and osteoinductive factors and could stimulate bone regeneration. J Orthop Res 22: 73-79, 2004.

23. Aho OM, Lehenkari P, Ristiniemi J, Lehtonen S, Risteli J and Leskelä HV: The mechanism of action of induced membranes in bone repair. J Bone Joint Surg Am 95: 597-604, 2013.

24. Marais LC and Ferreira N: Bone transport through an induced membrane in the management of tibial bone defects resulting from chronic osteomyelitis. Strateg Trauma Limb Reconstr 10: 27-33, 2015.

25. Marais LC, Ferreira N, Aldous C and Le Roux TL: The outcome of treatment of chronic osteomyelitis according to an integrated approach. Strateg Trauma Limb Reconstr 11: 135-142, 2016.

26. Dzyuba GG, Reznik LB, Erofeev SA and Odarchenko DI: Efficiency of local cement reinforcing antibacterial implants in surgical treatment of long bones chronic osteomyelitis. Khirurgiia (Mosk) 5: 31-36, 2016 (In Russian).

27. Karger C, Kishi T, Schneider L, Fitoussi F and Masquelet AC; French Society of Orthopaedic Surgery and Traumatology (SoFCOT): Treatment of posttraumatic bone defects by the induced membrane technique. Orthop Traumatol Surg Res 98: 97-102, 2012.

This work is licensed under a Creative Commons Attribution-NonCommercial-NoDerivatives 4.0 International (CC BY-NC-ND 4.0) License. 